\title{
ON LEBESGUE SUMMABILITY FOR DOUBLE SERIES
}

\author{
M. J. KOHN 1
}

ABSTRACT. In [2] a two dimensional analogue of Lebesgue's theorem on differentiation of formally integrated trigonometric series was established. Here we show that a stronger analogue holds.

1. Let $T: \sum_{n \in \mathbf{Z}} c_{n} e^{i n \theta}$ be a trigonometric series in one variable, with $c_{n} \rightarrow 0$. Let

$$
\lambda(\theta)=c_{0} \theta+\sum_{n \neq 0} \frac{c_{n}}{i n} e^{i n \theta}
$$

We say $T$ is Lebesgue summable at $\theta_{0}$ to sum $s$ if $\lambda(\theta)$ has at $\theta_{0}$ a first symmetric derivative with value $s$. That is, if $\frac{1}{2}\left\{\lambda\left(\theta_{0}+t\right)-\lambda\left(\theta_{0}-t\right)\right\}=s t+o(t)$ as $t$ $\rightarrow 0$. The following result is well known (see [3, p. 322]).

Theorem A. Suppose $c_{n}=O(1 / n)$ as $n \rightarrow \infty$ and suppose $T$ converges at $\theta_{0}$ to finite sum $s$. Then $T$ is Lebesgue summable at $\theta_{0}$ to $s$.

2. We are concerned here with a two dimensional analogue of Theorem $A$ for spherically convergent series. We denote points of $T_{2}$ by $x=\left(x_{1}, x_{2}\right)$ $=t e^{i \theta}$ and integral lattice points by $n=\left(n_{1}, n_{2}\right)$. We write $n \cdot x=n_{1} x_{1}$ $+n_{2} x_{2}$ and $|n|=\sqrt{n \cdot n}$.

Let $L(x)$ be defined in a neighborhood of $x_{0} \in T_{2}$. We will say, see [2], that $L$ has at $x_{0}$ a generalized first symmetric derivative with value $s$ if $L(x)$ is integrable over each circle $\left|x-x_{0}\right|=t$, for $t$ small, and if

$$
\frac{1}{2 \pi} \int_{0}^{2 \pi} L\left(x_{0}+t e^{i \theta}\right)(\cos \theta+\sin \theta) d \theta=\frac{1}{2} s t+o(t)
$$

as $t \rightarrow 0$.

If the limit in (2.1) exists only as $t$ tends to 0 through a set having 0 as a point of density, we will say $L(x)$ has at $x_{0}$ a generalized first symmetric approximate derivative.

The following result was established in [2].

THEOREM B. Let $T: \sum_{n \in \mathbf{Z}_{2}} c_{n} e^{i n \cdot x}$ be a double trigonometric series which converges spherically at $x_{0}$ to $s,|s|<\infty$. Suppose the coefficients of $T$ satisfy

Received by the editors January 5, 1976.

AMS (MOS) subject classifications (1970). Primary 42A92, 42A48, 42A24, 26A54.

Key words and phrases. Double trigonometric series, spherically convergent series, symmetric derivative, symmetric approximate derivative, Lebesgue summability.

${ }^{1}$ Partially supported by CUNY Faculty Research Award Program.

C American Mathematical Society 1976 


$$
\sum_{n_{1}+n_{2}=0}|n|^{\alpha}\left|c_{n}\right|^{2}+\sum_{n_{1}+n_{2} \neq 0}|n|^{\alpha}\left(n_{1}+n_{2}\right)^{-2}\left|c_{n}\right|^{2}<\infty
$$

for some number $\alpha>1$. Then the series

$$
\sum_{n_{1}+n_{2}=0} \frac{1}{2}\left(x_{1}+x_{2}\right) c_{n} e^{i n \cdot x}+\sum_{n_{1}+n_{2} \neq 0} \frac{-i c_{n}}{n_{1}+n_{2}} e^{i n \cdot x}
$$

converges spherically a.e. on $T_{2}$ to a function $L(x)$ which has at $x_{0}$ a generalized first symmetric approximate derivative equal to $s$.

3. In this paper we improve Theorem B by eliminating the word "approximate" from its conclusion. Thus we attain a closer analogue to Theorem A. Our result is

Theorem C. Let $T: \sum_{n \in \mathbf{Z}_{2}} c_{n} e^{i n \cdot x}$ be a double trigonometric series which converges spherically at $x_{0}$ to $s,|s|<\infty$. Suppose the coefficients of $T$ satisfy (2.2) for some number $\alpha>1$. Then the series (2.3) converges spherically a.e. on $T_{2}$ to a function $L(x)$ which has at $x_{0}$ a generalized first symmetric derivative with value $s$.

4. Proof of Theorem C. We may assume $x_{0}=0$, and $c_{0}=s=0$. Write $S_{u}=S_{u}(0)=\sum_{|n|<u} c_{n}$. Let

$$
g_{n}(x)= \begin{cases}\frac{-i}{n_{1}+n_{2}} e^{i n \cdot x} & \text { if } n_{1}+n_{2} \neq 0 \\ \frac{1}{2}\left(x_{1}+x_{2}\right) e^{i n \cdot x} & \text { if } n_{1}+n_{2}=0\end{cases}
$$

Then, for $n \neq 0$, by the Lemma of [2],

$$
\frac{1}{2 \pi} \int_{0}^{2 \pi} g_{n}\left(t e^{i \theta}\right)(\cos \theta+\sin \theta) d \theta=\frac{J_{1}(|n| t)}{|n|} .
$$

Let

$$
L_{R}(x) \sum_{\substack{|n|<R \\ n_{1}+n_{2}=0}} \frac{1}{2}\left(x_{1}+x_{2}\right) c_{n} e^{i n \cdot x}+\sum_{\substack{|n|<R \\ n_{1}+n_{2} \neq 0}} \frac{-i c_{n}}{n_{1}+n_{2}} e^{i n \cdot x} .
$$

The condition (2.2) on $c_{n}$ insures that $L(x)=\lim _{R \rightarrow \infty} L_{R}(x)$ exists a.e. on each circle $|x|=t$. This is a consequence of Theorem 1 of [1]. Moreover, by Theorem 2 of [1],

$$
\int_{0}^{2 \pi} \sup _{R}\left|L_{R}\left(t e^{i \theta}\right)\right| d \theta<\infty
$$

so we may integrate the series defining $L(x)$ term by term over each circle. Thus, 


$$
\begin{aligned}
\frac{1}{2 \pi} \int_{0}^{2 \pi} L\left(t e^{i \theta}\right) & (\cos \theta+\sin \theta) d \theta \\
& =\frac{1}{2 \pi} \int_{0}^{2 \pi} \lim _{R \rightarrow \infty} \sum_{|n|<R} c_{n} g_{n}\left(t e^{i \theta}\right)(\cos \theta+\sin \theta) d \theta \\
& =\lim _{R \rightarrow \infty} \sum_{|n|<R} c_{n} \frac{1}{2 \pi} \int_{0}^{2 \pi} g_{n}\left(t e^{i \theta}\right)(\cos \theta+\sin \theta) d \theta \\
& =\lim _{R \rightarrow \infty} \sum_{|n|<R} c_{n}|n|^{-1} J_{1}(|n| t) \\
& =\lim _{R \rightarrow \infty} t \sum_{|n|<R} c_{n} \gamma(|n| t),
\end{aligned}
$$

where $\gamma(z)=z^{-1} J_{1}(z)$

We express the last sum as an integral and integrate by parts.

$$
\sum_{|n|<R} c_{n} \gamma(|n| t)=S_{R} \gamma(R t)-\int_{0}^{R} S_{u} \frac{d}{d u} \gamma(u t) d u .
$$

Since the series $T$ is spherically convergent to 0 at $x_{0}=0$ and since $J_{1}(z)=O\left(z^{-1 / 2}\right)$ as $z \rightarrow \infty$,

$$
S_{R} \gamma(R t)=o(1) O\left(R^{-3 / 2}\right)=o(1)
$$

as $R \rightarrow \infty$. Therefore, returning to (4.1)

$$
\begin{aligned}
\frac{1}{2 \pi} \int_{0}^{2 \pi} L\left(t e^{i \theta}\right) & (\cos \theta+\sin \theta) d \theta \\
= & \lim _{R \rightarrow \infty} t \sum_{|n|<R} c_{n} \gamma(|n| t) \\
= & -t \int_{0}^{\infty} S_{u} \frac{d}{d u} \gamma(u t) d u \\
& =-t\left\{\int_{0}^{1 / t} S_{u} \frac{d}{d u} \gamma(u t) d u+\int_{1 / t}^{\infty} S_{u} \frac{d}{d u} \gamma(u t) d u\right\} \\
& =-t\{A(t)+B(t)\} .
\end{aligned}
$$

We will show $A(t)$ and $B(t)$ each tend to 0 with $t$. To estimate $A(t)$ we note that $\gamma(z)$ is an entire function, so for $|z|<1,\left|\gamma^{\prime}(z)\right| \leqslant K$.

$$
A(t)=\int_{0}^{1 / t} o(1) \cdot t K d u=o(1)
$$

To estimate $B$ we note

$$
\frac{d}{d z} \gamma(z)=\frac{d}{d z}\left(z^{-1} J_{1}(z)\right)=-z^{-1} J_{2}(z)=z^{-1} O\left(z^{-1 / 2}\right)=O\left(z^{-3 / 2}\right) .
$$

Thus 


$$
B(t)=\int_{1 / t}^{\infty} o(1) \cdot t O(u t)^{-3 / 2} d u=o(1)
$$

This completes the proof of Theorem C.

\section{BIBLIOGRAPHY}

1. M. Kohn, Spherical convergence and integrability of multiple trigonometric series on hypersurfaces, Studia Math. 44 (1972), 345-354. MR 49 \#9535.

2. - Lebesgue summability of double trigonometric series, Trans. Amer. Math. Soc. (to appear).

3. A. Zygmund, Trigonometric series, Vol. I, 2nd rev. ed. reprint, Cambridge Univ. Press, New York, 1968. MR 38 \#882.

Department of Mathematics, Brooklyn College, Brooklyn, New York 11210 